\title{
A three-dimensional fluid-structure interaction (FSI) model for estimating the heart energy consumption of blood flow through the aortic valve
}

\author{
Ali Vazifehdoostsaleh ${ }^{a^{*}}$, Morteza Darjani ${ }^{\mathrm{a}}$ and Aram Bahmani ${ }^{\mathrm{b}, \mathrm{c}}$
}

${ }^{a}$ Department of Mechanical Engineering, Ramsar Branch, Islamic Azad University, Ramsar, Iran

${ }^{b}$ Department of Mechanical Engineering, McGill University, Montreal, QC H3A 2K6, Canada

${ }^{c}$ Department of Mechanical and Mechatronics Engineering, University of Waterloo, 200 University Ave. West, Waterloo N2L 3G1, Canada

\begin{tabular}{l}
\hline A R T I C L EI N F O \\
\hline Article history: \\
Received 15 September 2019 \\
Accepted 6 November 2019 \\
Available online \\
6 November 2019 \\
\hline Keywords: \\
Cardiac output \\
Echo-Doppler flow \\
Fluid- structure interaction \\
Stroke volume
\end{tabular}
\begin{abstract}
A B S T R A C T
The cardiac output and stroke volume of a healthy person was estimated using a 3D fluid-structure interaction (FSI) simulation coupled with an echocardiogram Doppler (echo-Doppler) during exercising and resting. The geometry, dimensions, and blood flow through the aortic valve were measured using the echo-Doppler. A 3D FSI simulation was modeled using an arbitrary Lagrangian-Eulerian mesh. The 3D FSI cardiac output and stroke volume results were $15.4 \%$ lower than Doppler results when brachial pressures applied with differences between brachial, central, and left ventricular pressures. While without considering these brachial pressures differences, the discrepancy between the FSI cardiac output and stroke volume results with Doppler increased to $22.3 \%$ and $26.2 \%$, respectively. Eventually, the comparisons of the 3D FSI results and clinical measurements demonstrated that numerical methods can be a potential computational tool to estimate cardiac output and stroke volume for different heart rates when they coupled with the clinical measurements.
\end{abstract}

\section{Introduction}

Cardiac diseases still are one of the significant causes of death (Murphy \& Xu, 2012). Cardiac outputs can be a crucial parameter during the developing diagnosis and treatments of cardiovascular diseases (Criner et al. 2010). Heart failure can be an exemplary failure of the heart to maintain a cardiac output which leads to body metabolism. Hence, for the purpose of diagnosis these types of diseases, determining the volume of stroke and evaluating blood pumping function would be efficacious and significant. There are several invasive techniques which utilized to determine either cardiac output or volume of stroke. However, these procedures have complicated, expensive, and risky processes (Lavdaniti 2008). In order to decrease the requirements of using invasive procedures, computational modeling can be potential and have a remarkable contribution to measuring cardiac outputs and stroke volume. A number of clinical methods are used to measure cardiac output which is invasive or expensive such as ultrasound, magnetic resonance imaging, and angiography catheterization (Engoren and Barbee 2005; Hofer et al. 2007; Lavdaniti 2008). Cardiac output and volume of stroke are measured using breath-by-breath oxygen and released carbon monoxide during cycling via Knobloch et al. (2008). In Knobloch et al. (2008) study,

* Corresponding author.

E-mail addresses: Alivazifedoost@gmail.com (A. Vazifehdoostsaleh) 
non-invasive ultrasound-Doppler imaging on healthy adult athletes was employed. Also, they found that there are correlations among cardiac output, cardiac index, heart rate, the volume of stroke, and consumed O2. Sugawara et al. (2003) attempted to determine cardiac output by means of a particular model-flow method. They compared their results with some data which were from echocardiogram Doppler and found that their proposed model could be more accurate than echocardiogram Doppler. Stringer et al. (1997) proposed a particular formula for non-invasive hemodynamics in exercise testing. Based on the Stringer et al. (1997) study, Knobloch et al. (2009) compared Stringer's formula with other clinical methods called ultrasound cardiac output monitoring which both of them were utilized for calculation of cardiac output. Likewise, three miscellaneous techniques were conducted by Christie et al. (1987) in order to measure and compare cardiac output. Moreover, Maroni et al. (1998) employed a first-pass radionuclide ventriculographic technique to diagnose myocardial dysfunction and also, to determine cardiac output. This technique was a non-invasive and inexpensive one; however, it is not available now due to detrimental effects during the measurement of cardiac outputs. Among mentioned techniques, the computational method can be applied to predict cardiac outputs. Aortic and heart valves are occurred and altered via blood flow; hence, fluid-structure interaction (FSI) modeling can be the applicable method (Caro et al. 1978, Bellhouse 1972). In the FSI modeling, reaction forces and stresses due to fluid pressure in solid phase can be measured (Dowell \& Hall, 2001; Van de Vosse et al., 2003; Wall et al., 2006). In this technique, an arbitrary Lagrange-Euler (ALE) mesh is utilized in order to solve and determine not only stresses and deformations of structure phase by means of finite element solutions but also the behavior of fluid by the aid of computational fluid dynamics (Donea et al., 1982; Formaggia \& Nobile, 1999). During the past decade, FSI has been employed to investigate the mechanical and biological behavior of heart valves (Al-Atabi et al., 2010; Espino et al., 2014, 2015, Stijnen et al., 2004; Xia et al., 2005). For instance, regarding heat simulation, De Hart et al. (2000, 2003a, 2003b) performed a 2D and 3D FSI model and also, they assigned a particular fiber reinforced composites for leaflets. Indeed, the feasibility of developing complicated aortic valve models has been indicated. However, combining noninvasive clinical calculations and prediction, heart rate has been less considered in predicting the cardiac output of patients. In this paper, a 3D FSI aortic valve model is simulated to measure either cardiac output or stroke volume during exercise. Moreover, the natural aortic valve is simulated, to exemplify, by pumping blood flow and increasing systolic pressure through aortic artery valve is opened, and then blood flow is ejected after that ventricular systolic pressure tends to be decreased. Consequently, aortic valve comes to closure. It should be noted that systolic pressure and boundary conditions of the model were applied, based on brachial pressure measurement. Hence, different parameters such as cardiac output, stroke volume, peak and mean of velocity, and velocity-time integration can be determined via combining FSI simulation and the data of non-invasive measurements. The 3D geometry and boundary conditions were performed according to a volunteer; therefore, these predictions belong to a particular volunteer. The model would be validated by comparing echocardiography (ECG) data and also, mechanical properties are assigned based on literature information.

\section{Combination of clinical method with numerical modeling}

\subsection{Experimental design}

During exercise and rest time, the hemodynamic data of a 33 years old male was recorded. These data would be compared with FSI results. Based on protocols which are approved via the department of cardiovascular Imaging (Shaheed Rajaei Cardiovascular, Medical, and Research Center, Tehran, Iran), informed consent was obtained from the participant. According to maximal cycling and Doppler ECG, we found that participant has normal cardiovascular performance. Moreover, exercise composed of participant pedaling on the bicycle, with required images of blood flow through the aortic valve which is measured by heart's five-chamber view in climax area by means of B-mode. A particular brachial pressure recorder was utilized which was fixed on the left arm. Regarding the regimes of exercising, the rate of participant's heart was raised to approximately $180 \mathrm{bpm}$ via maximal cycling tests. 


\subsection{Cardiovascular measurements}

A commercial ultrasonography Maylab-desk software was employed to examine the ECG. For the purpose of recording blood flow through the aortic valve, a $4 \mathrm{MHz}$ phased-array probe was installed at the climax in order to observe heart's five-chamber view. The geometry of aortic valve was estimated via a transducer which is located at heart's three-chamber view. Furthermore, blood flow was measured via an echo-Doppler flow at miscellaneous heart rates from rest to maximal cycling test. In order to capture high-resolution images with the ECG, a particular chair was utilized, and volunteer body was fixed completely to the chair. By means of Maylab-desk analyzer, high-resolution Echo-Doppler images were saved digitally and analyzed. Fig.1 indicates either systolic or diastolic pressure of the brachial artery during rest and cycling time. Moreover, obtained ventricular, brachial, and central pressures are illustrated in this Figure.

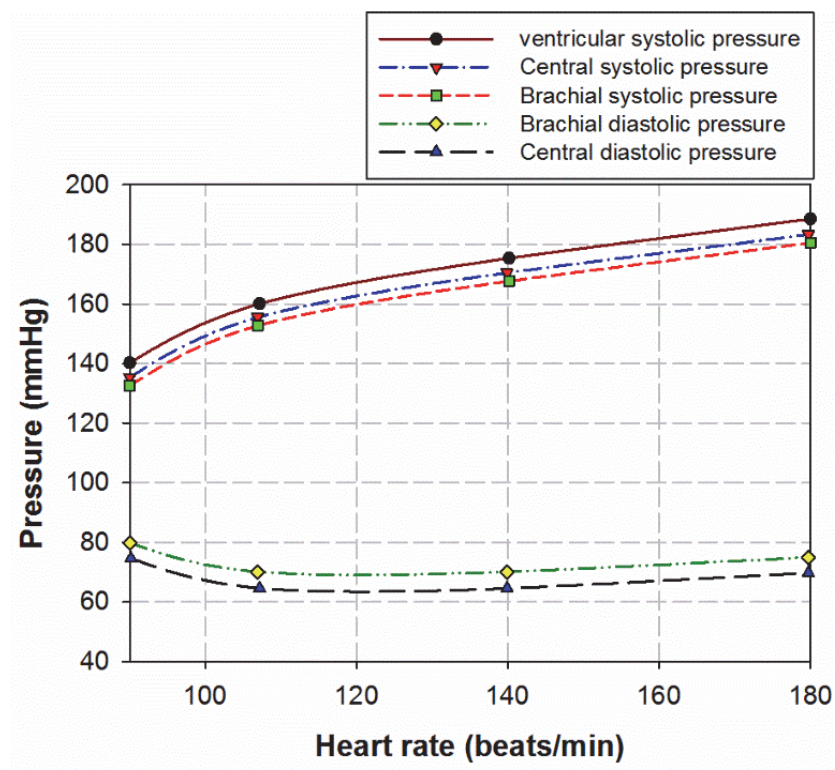

Fig.1. Interpolated curves for brachial systolic and diastolic pressures, central systolic and diastolic pressures, and ventricular systolic pressure versus heart rate.

In order to calculate central pressure from brachial pressure data, Eq. (1) and Eq. (2) were utilized.

Central systolic pressure $\approx$ brachial systolic pressure +2.25 ,

Central diastolic pressure $\approx$ brachial diastolic pressure -5.45 ,

where all pressures were obtained in $\mathrm{mmHg}$.

Park et al. (2011) compared brachial pressure acquired via Oscillometry with central pressure acquired by an invasive method, and they could drive these equations. In this study, left ventricular and central systolic pressure were measured. the difference value between the peak left ventricular and central systolic pressure were reported by Laske et al. (1996) which was approximately $5 \mathrm{mmHg}$. It is worth mentioning, one of the significant parameters in plotting ventricular systolic pressure is ejection time. In effect, by means of the Doppler-flow images under B-mode, ECG data, and Maylab-desk software, the time of ejection was measured. This process was conducted via tracing Doppler flow in a larger region and more regular border. In order to measure heart rate, ejection time, left ventricular systolic pressure, and central diastolic pressure, a general waveform of left ventricle pressure during ejection time is required which is reported by Guyton and Hall (1996) and demonstrated in Fig. 2. We also extracted these parameters from the scanned plot of our data based on this general waveform. 


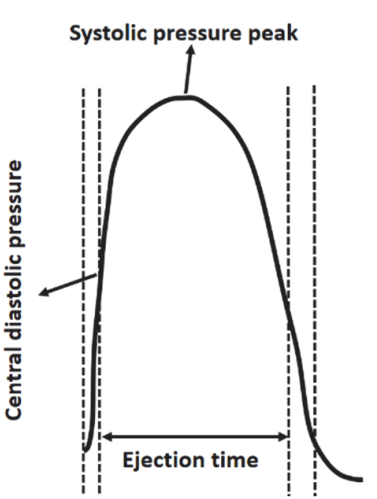

Fig. 2. Tracings ejection time, systolic pressure peak, and central diastolic pressure of the left ventricular general waveform

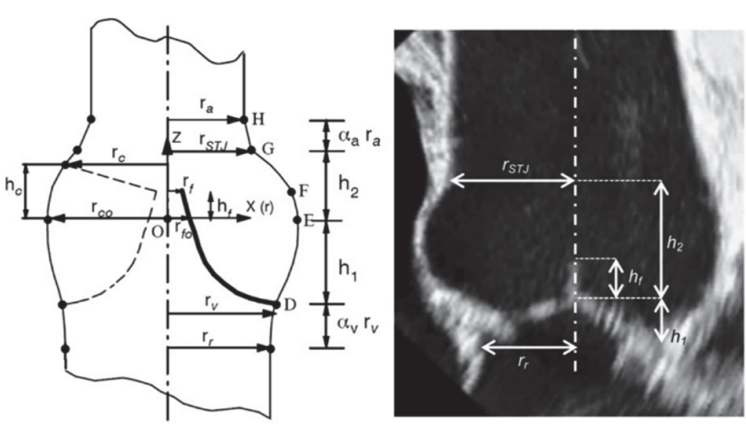

Fig. 3. Parametric dimensions and ECG imaging of aortic valves and leaflets

\subsection{Fluid structure interaction}

Fig.3 illustrates the parametric dimensions and clinical imaging of aortic valves and leaflets. According to data extracted from T-wave of ECG in the maximum opening area, a 3D geometry of aortic root and chamber of aortic sinus valsalva was generated by Solid works software. The diameters of the aortic valve annulus and sinus valsalva were obtained at climax $\mathrm{T}$-wave time utilizing a resting parasternal long-axis view. All dimensions of the 3D model are indicated in Table 1 and Fig. 3. Clark and Finke (1974) found that the thickness of heart valve leaflets is not uniform; however, a uniform thickness $0.5 \mathrm{~mm}$ has been considered in our model.

Table 1. The parametric and calibrated dimensions of the aortic valves and leaflets geometry used in the 3D model

\begin{tabular}{ccc}
\hline & Parametric dimensions reported in the literature & Calibrated dimensions used in the 3D model \\
\hline$r_{r}$ & $12 \mathrm{~mm}$ & $12 \mathrm{~mm}$ \\
$r_{a}$ & $1.1 r_{r}$ & $13.75 \mathrm{~mm}$ \\
$r_{s}$ & $1.4 r_{r}$ & $15.75 \mathrm{~mm}$ \\
$\mathrm{~h}_{1}+h_{2}$ & $(0.6+0.8) \mathrm{r}_{r}=1.4 r_{r}$ & $17 \mathrm{~mm}$ \\
$\mathrm{~h}_{f}$ & $0.35 r_{r}$ & $6.3 \mathrm{~mm}$ \\
$\mathrm{~h}_{c}$ & $0.5 r_{r}$ & $11 \mathrm{~mm}$ \\
$r_{f_{0}}$ & $0.27 r_{r}$ & $0.01 r_{r}$ \\
$r_{f}$ & $0.07 r_{r}$ & $0.01 r_{r}$ \\
$n$ & 1.5 & 1.1 \\
$N$ & 1.2 & 1.1 \\
$m$ & 1.25 & 1.25 \\
\hline
\end{tabular}

The material properties of leaflets and blood have been indicated in Table 2 and were evaluated from literature (Govindarajan et al., 2010; Koch et al., 2010). According to this table, an isotropic linear behavior was selected for leaflets. Furthermore, an incompressible and Newtonian behavior was considered for blood. In fact, Caro et al. (1978) reported these types of blood behavior through left ventricle out towards aorta.

Table. 2. The mechanical properties of leaflets and blood

\begin{tabular}{cccc} 
Viscosity $(\mathrm{mPa}-\mathrm{s})$ & Young's modulus $(\mathrm{MPa})$ & Poisson's ratio & Density $\left(\mathrm{kg} / \mathrm{m}^{3}\right)$ \\
\hline 4 & 4 & 0.45 & 1050 \\
\hline
\end{tabular}


Fig. 4 illustrates applied left ventricular pressures for miscellaneous heart rates. In fact, at inflow boundary of the aortic root in left ventricular, the pressure was applied. It should be noted that by increasing heart rate, peak pressure tends to be soaring; however, peak time tends to be declined.

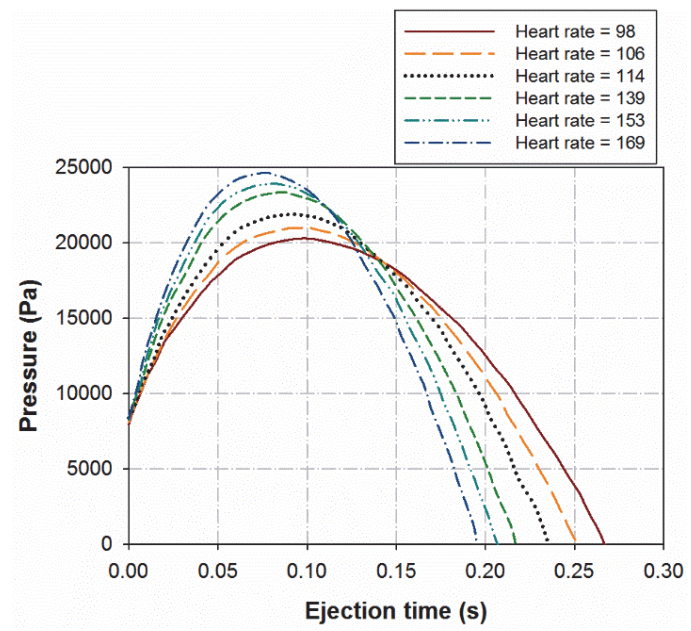

Fig. 4. Pressure curves of the left ventricle versus ejection time in various heart rates.

Based on Fig.1, central diastolic pressure depends on heart rate; therefore, it was applied at outflow boundary of the aortic heart valve which is shown in Fig.5. The rigid boundaries and no-slip behavior were selected for aorta walls. By means of Eq. (3), the velocity of shared and contacted boundaries between valve leaflets and fluid domain can be defined, which is equivalent to the velocity of valve leaflets.

$$
u=\frac{\partial x}{\partial t}, v=\frac{\partial y}{\partial t}
$$

where $u$ and $v$ represent the $\mathrm{X}$ - and Y-axis velocities, respectively. Moreover, $\frac{\partial x}{\partial t}$ and $\frac{\partial y}{\partial t}$ represent time-dependent displacement through the $\mathrm{X}$-axis and $\mathrm{Y}$-axis. It should be noted that the Cartesian coordinate system had been defined in two orthogonal axes for X-and Y-axis. Fig.3. demonstrates the $\mathrm{Y}$-axis which is parallel to both inflow and outflow aorta boundaries, and also represent the $\mathrm{X}$-axis that is perpendicular to them. Besides, Fig.5 indicates the leaflets' boundaries which were restricted in their aortic wall. Likewise, forces were applied via fluid dynamics on the leaflets' boundaries and result in valve deflecting. For the purpose of solving FSI problems, several couplings are required. For instance, by means of Eq.(3) fluid flow and structural deformation are coupled via a velocity constraint. In addition, equal reaction forces are produced via fluid loads subjected to the structure. In a simultaneous interaction (i.e., FSI) a two-way coupling can be produced by aforementioned constraints. Lagrange multipliers can be calculated via a non-ideal formulation from fluid dynamics which are equivalent to fluid forces. Thus, loading conditions can be defined via Eq.(4). In fact, by considering full stress tensor, Newtonian, and laminar flow, and continuity and incompressible Navier -Stokes equations fluid dynamics can be solved. (Espino et al., 2015)

$$
(\sigma . n)_{\text {Fluid }}=(\sigma . n)_{\text {Solid }}
$$

where $\sigma$ and $n$ represent stress tensor and normal vector to FSI boundary respectively.

Fig. 5 also indicates a moving mesh technique which is well-known as ALE was employed by means of HYPERMESH software. In this technique, for the solid or structural domain, a Lagrangian framework is provided, in terms of fluid domain, an Eulerian framework is enabled as well. Hence, fluid mesh deformations can be explored via this technique and other boundaries fixed completely. Commercial 
explicit finite element solver LS-DYNA was employed in order to solve FSI model under time-dependent conditions. FSI was modeled through penalty coupling method available in LS-DYNA software. Algorithms were applied to both structural and fluid domains to prevent hourglass effect. Mesh discrepancy between Lagrangian and Eulerian parts assumed the ideal 1:1 ratio in order to achieve better results in penalty coupling method. The mesh of three-dimensional blood flow domain in deformable leaflet valve model within applied boundary conditions.
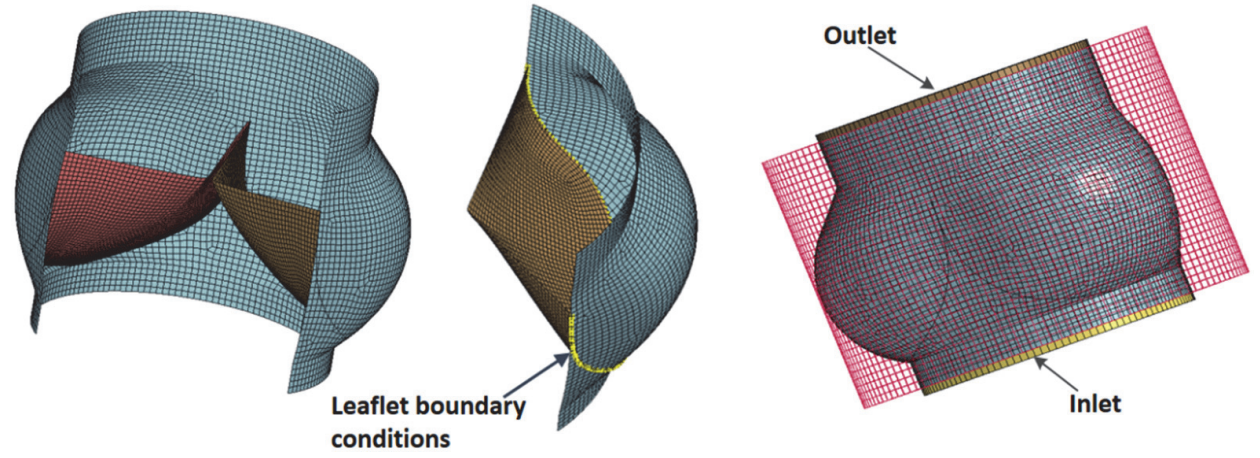

Fig. 5. ALE mesh and boundary conditions applied for 3D FSI model

Fig. 6. (a) and (b) represent mesh convergence with three different element sizes for solid and fluid domains. For coarse elements with a range of 0.4-0.9 mm, 96000 fluid elements and 15557 solid elements were used. For medium size elements with a range of 0.3-0.7 mm, 142578 fluid elements and 23870 solid elements were considered. For diminutive size elements with the range of 0.2-0.5 mm, 203422 fluid elements and 35926 solid elements were used.

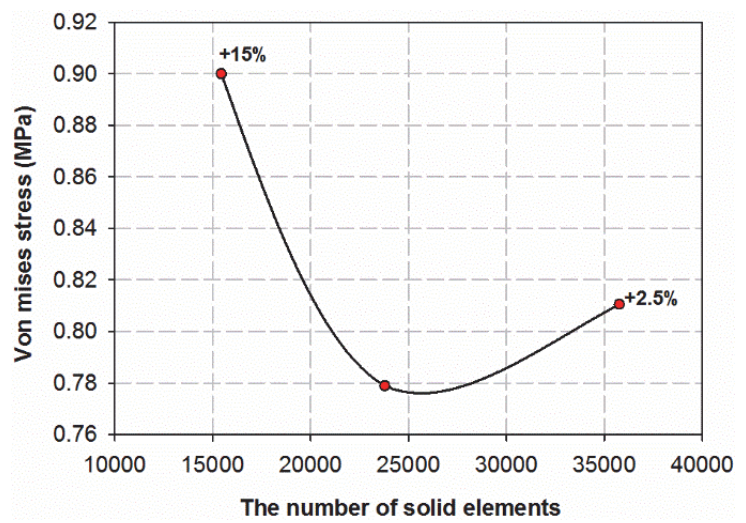

(a)

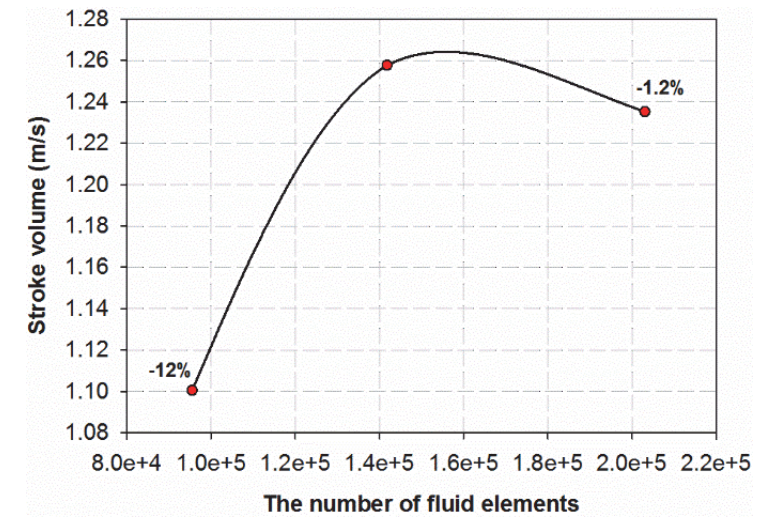

(b)

Fig. 6. The number of fluid and solid elements for a mesh independent 3D FSI model

In order to calculate the cardiac output, the Eq. (5) can be used

Cardiac output $=$ Stroke volume $\times$ Heart rate,

where by using ECG data, stroke volume in Eq. (5) was determined via Eq. (6)

Stroke volume $=$ Velocity integration $\times$ Aortic area,

also, by tracing Doppler flow captured by ultrasound imaging, velocity integration in Eq. (6) was obtained. Aortic area can be expressed as follow,

$$
\text { Area }=\pi\left(\frac{D}{2}\right)^{2} \text {, }
$$


where $D$ is obtained ascending aortic diameter after sinotubular junction.

Figs. (8a), and (8b) represents the average of velocity which was computed at any time step of ejection cycle via Eq. (8)

$$
\text { Velocity integration }=\oint_{0}^{\text {Ejection time }} V . d t \text {, }
$$

where $V$ is the velocity of fluid through outlet boundaries. Either stoke volume or cardiac output were computed and compared with echo-Doppler data. It should be mentioned that average velocity for any heart rate was measured by means of Eq. (9),

$$
\text { Average velocity }=\frac{\text { Velocity integration }}{\text { Ejection time }}
$$

In effect, comparison the average velocity, cardiac output, and stroke volume enabled quantitative validation of the FSI simulation.

\section{Results and discussion}

In this section, first, the mesh indecency and convergence of numerical results computed using the FSI model outlined in Section 5 are indicated. Afterward, the deformations and timing of aortic and leaflet valves simulated using the FSI model are shown. Then, the visualized blood hydrodynamic results, as well as, a comparison between numerical and clinical predictions are presented and discussed.

\subsection{Aortic valves and leaflets dynamics}

Fig.7 shows the displacement contours of aortic valves and leaflets during ejection time in a full cycle of systole and diastole in eight-time intervals (i.e., four intervals for each period). The maximum opening value of leaflets is $60.78 \%$ of the aortic valves cross-section area which is very close to the values reported in the literature (De Hart et al., 2003b). In addition, the waveform motion of leaflets in systole and diastole at each time interval can be seen in Fig.7. These observations were also reported in the literature for each time intervals showed in this Figure (De Hart et al., 2003a and 2003b; Carmody et al., 2006). Moreover, the clinical observations reported that the maximum opening of aortic valves and leaflets has occurred in the systole phase when ejection time is $140 \mathrm{~ms}$. It can be easily seen in this Figure that the maximum opening of aortic valves and leaflets in the FSI model is the same as clinical observations.

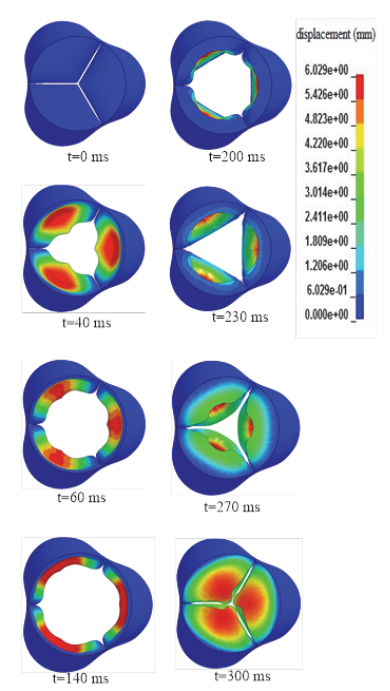

Fig.7. Deformations and morphologies of leaflets during a complete cycle of ejection. Left column is the systole phase, and right column is the diastole phase. 


\subsection{Hydrodynamics of blood}

The pressure contours during ejection time in a full cycle of systole and diastole in six-time intervals demonstrated in Fig 8. As it can be seen in this Figure, before fully opening aortic valves and leaflets, the pressure differences in both side of valves are higher due to the effects leaflets in the aortic valves. Note that the leaflets and aortic valves are started to opening under a minimal pressure difference. Although there is a notable inverse pressure in the diastole phase, the leaflets prevent the jet flow toward the ventricle.

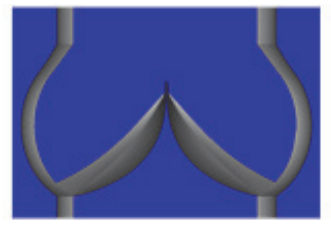

$\mathrm{t}=0 \mathrm{~ms}$

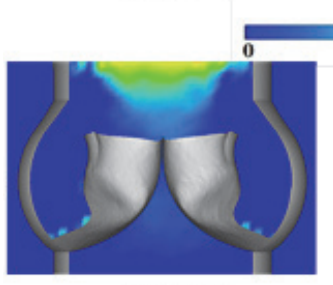

$\mathrm{t}=220 \mathrm{~ms}$

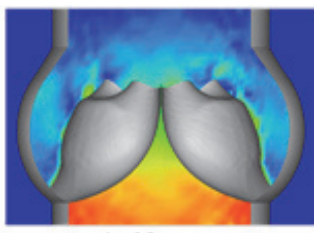

$\mathrm{t}=40 \mathrm{~ms}$

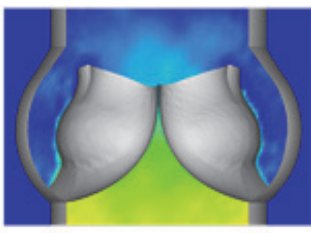

$\mathrm{t}=110 \mathrm{~ms}$ [mmHg]
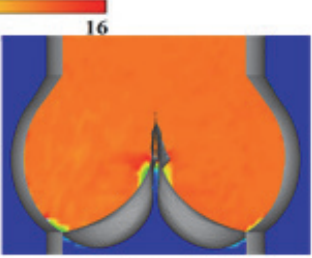

$\mathrm{t}=300 \mathrm{~ms}$

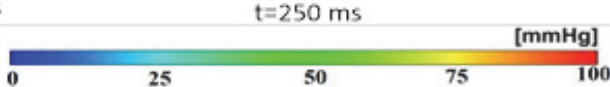

Fig. 8. Pressure contours during a full cycle of ejection for systole and diastole phase

Fig. 9 also shows the velocity contours of blood during ejection time in a full cycle of systole and diastole in six-time intervals. The maximum value of blood velocity is $1.25 \mathrm{~m} / \mathrm{s}$ occurred at $\mathrm{t}=110 \mathrm{~ms}$; while the clinical measurements reported $1.34 \pm 0.34 \mathrm{~m} / \mathrm{s}$ for this value at the same time interval. In the closing phase, by increasing pressure at the aortic side and decreasing pressure at the ventricle side, the jet flow confronts with a pressure resistance; thus, jet flows are redirected toward to sinuses. These vortexes in the sinuses which are occurred $\mathrm{t}=220$ and $300 \mathrm{~ms}$ have a crucial influence on quickly and correctly closing leaflets.

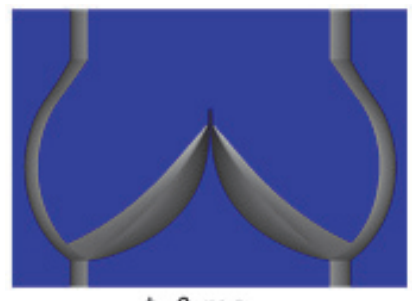

$\mathrm{t}=0 \mathrm{~ms}$

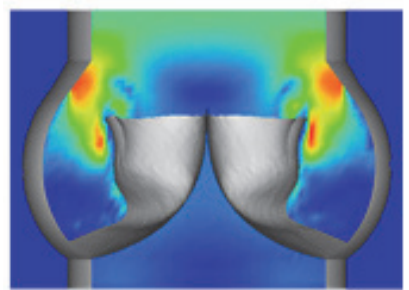

$\mathrm{t}=220 \mathrm{~ms}$

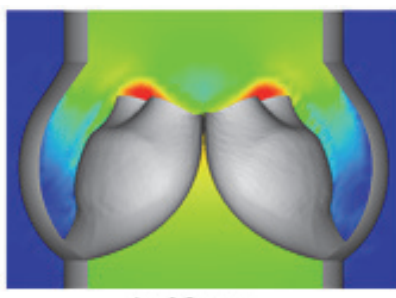

$\mathrm{t}=40 \mathrm{~ms}$

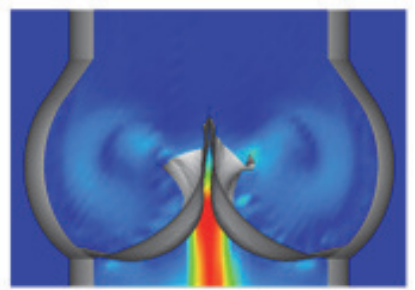

$\mathrm{t}=250 \mathrm{~ms}$

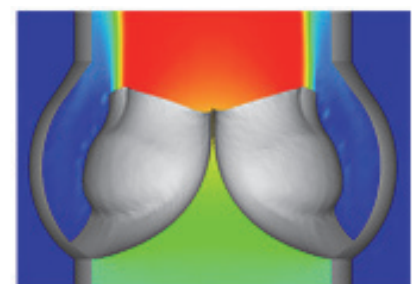

$\mathrm{t}=110 \mathrm{~ms}$

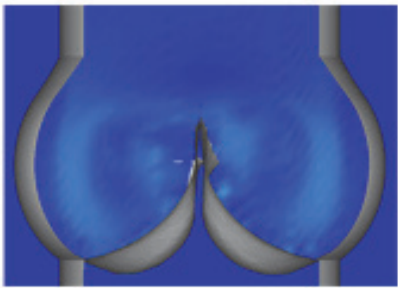

$\mathrm{t}=300 \mathrm{~ms}$

[m/s]

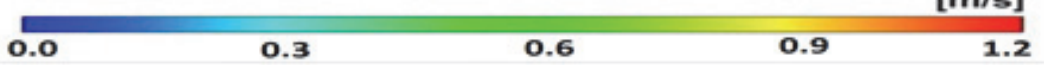

Fig.9. Velocity contours during a full cycle of ejection for systole and diastole phase 


\subsection{Comparison of numerical results and clinical measurements}

Table 3 and 4 present 3D FSI simulation predictions and echo-Doppler data. The peak blood flow velocity through aorta increased by $16.6 \%$ from 98 to $147 \mathrm{bpm}$ and increased a further $2.3 \%$ as the heart rate increased to $169 \mathrm{bpm}$. The mean velocity predicted by 3D FSI simulations was on average $14.8 \%$ lower than echo-Doppler measurements (i.e. an average for the whole protocol) when pressure differences were accounted for. This difference increased to $22.4 \%$ when the pressure differences were not accounted. The cardiac output predicted by 3D FSI simulation was on average $15.4 \%$ lower when compared to echo-Doppler results, for the whole of the protocol, when pressure differences were accounted for. The corresponding difference, when pressure differences were excluded, was $22.3 \%$. Three-dimensional FSI simulations that accounted for pressure differences predicted a stroke volume that was on average $15.3 \%$ lower than that derived from echo-Doppler. This increased to $26.2 \%$ when pressure differences were ignored. For FSI simulations, the mean velocity increased by $15.7 \%$ as the heart rate increased from 98 to $136 \mathrm{bpm}$ when pressure differences were accounted for and increased by $4.6 \%$ from a heart rate of 136 to $169 \mathrm{bpm}$. When pressure differences were accounted for, FSI simulations showed a $2.9 \%$ increase in stroke volume from a heart rate of $98-114 \mathrm{bpm}$. Then, this stopped from heart rates of approximately $136-147 \mathrm{bpm}$.

Table 3. Comparisons of echo-Doppler data with 3D FSI model results

\begin{tabular}{|c|c|c|c|c|c|c|c|c|c|c|c|}
\hline $\begin{array}{l}\text { HR } \\
(\mathrm{bpm})\end{array}$ & $\begin{array}{l}\mathrm{VSP} / \mathrm{CDP} \\
(\mathrm{mmHg})\end{array}$ & $\begin{array}{c}\mathrm{VSP}^{\mathrm{a}} / \mathrm{CDP}^{\mathrm{a}} \\
(\mathrm{mmHg})\end{array}$ & $\begin{array}{l}\text { VPD } \\
(\mathrm{m} / \mathrm{s})\end{array}$ & $\begin{array}{l}\mathrm{VPN} \\
(\mathrm{m} / \mathrm{s})\end{array}$ & $\begin{array}{l}\mathrm{VPN}^{\mathrm{a}} \\
(\mathrm{m} / \mathrm{s})\end{array}$ & $\begin{array}{l}\text { VMD } \\
(\mathrm{m} / \mathrm{s})\end{array}$ & $\begin{array}{l}\mathrm{VMN} \\
(\mathrm{m} / \mathrm{s})\end{array}$ & $\begin{array}{l}\mathrm{VMN}^{\mathrm{a}} \\
(\mathrm{m} / \mathrm{s})\end{array}$ & $\begin{array}{c}\text { COD } \\
(\mathrm{ml} / \mathrm{min})\end{array}$ & $\begin{array}{c}\mathrm{CON} \\
(\mathrm{ml} / \mathrm{min})\end{array}$ & $\begin{array}{c}\mathrm{CON}^{\mathrm{a}} \\
(\mathrm{ml} / \mathrm{min})\end{array}$ \\
\hline 98 & $152 / 68$ & $144 / 74$ & 1.49 & 1.51 & 1.36 & 1.05 & 0.89 & 0.79 & 11356 & 9884 & 8773 \\
\hline 106 & $158 / 65$ & $152 / 71$ & 1.50 & 1.59 & 1.48 & 1.10 & 0.95 & 0.86 & 12651 & 10864 & 9935 \\
\hline 114 & $165 / 63$ & $157 / 69$ & 1.58 & 1.67 & 1.54 & 1.11 & 1.00 & 0.90 & 14051 & 11829 & 10672 \\
\hline 125 & $169 / 63$ & $163 / 69$ & 1.60 & 1.70 & 1.59 & 1.21 & 1.03 & 0.94 & 15298 & 12884 & 11938 \\
\hline 136 & $174 / 64$ & $167 / 70$ & 1.79 & 1.74 & 1.62 & 1.24 & 1.03 & 0.95 & 16172 & 13518 & 12489 \\
\hline 147 & $178 / 65$ & $171 / 71$ & 1.58 & 1.76 & 1.65 & 1.25 & 1.05 & 0.97 & 17225 & 14600 & 13424 \\
\hline 153 & $180 / 66$ & $173 / 72$ & 1.74 & 1.77 & 1.66 & 1.24 & 1.03 & 0.97 & 17330 & 14625 & 13655 \\
\hline 159 & $182 / 67$ & $175 / 72$ & 1.77 & 1.78 & 1.68 & 1.26 & 1.06 & 0.97 & 17941 & 15108 & 13961 \\
\hline 169 & $186 / 68$ & $178 / 74$ & 1.63 & 1.80 & 1.68 & 1.28 & 1.08 & 0.98 & 18849 & 15832 & 14504 \\
\hline
\end{tabular}

Table 4. Comparison of stroke volume data measured by echo-Doppler with 3D FSI model results with and without conseidering the effects of valvular -arterial pressure differences

\begin{tabular}{|c|c|c|c|c|c|c|}
\hline $\begin{array}{l}\text { Heart rate } \\
(\mathrm{bpm})\end{array}$ & $\begin{array}{c}\text { SVD } \\
\text { (ml/beat) }\end{array}$ & $\begin{array}{c}\text { SVN } \\
\text { (ml/beat) }\end{array}$ & $\begin{array}{l}\mathrm{SVN}^{\mathrm{a}} \\
\text { (ml/beat) }\end{array}$ & $\begin{array}{c}\text { Sugawara et al. } \\
(2003) \\
(\mathrm{ml} / \text { beat })\end{array}$ & $\begin{array}{c}\text { Christie et al. } \\
\text { (1987) } \\
(\mathrm{ml} / \text { beat })\end{array}$ & $\begin{array}{l}\text { Percentage of } \\
\text { difference of } \\
\text { SVN to SVD }\end{array}$ \\
\hline 98 & 115.8 & 100.9 & 89.5 & 80 & 102.4 & 12.9 \\
\hline 106 & 119.4 & 102.5 & 93.7 & 83 & 108 & 14.1 \\
\hline 114 & 123.3 & 103.8 & 93.6 & 86 & 109.5 & 15.8 \\
\hline 125 & 122.4 & 103.1 & 95.5 & 89 & 105 & 15.8 \\
\hline 136 & 118.9 & 99.4 & 91.8 & 92 & 100.6 & 16.4 \\
\hline 147 & 117.2 & 99.3 & 91.3 & 94 & 100.5 & 15.2 \\
\hline 153 & 113.3 & 95.6 & 89.2 & $\mathrm{Na}$ & 102.5 & 15.6 \\
\hline 159 & 112.8 & 95 & 87.8 & $\mathrm{Na}$ & 103.6 & 15.8 \\
\hline 169 & 111.5 & 93.7 & 85.8 & $\mathrm{Na}$ & 104.7 & 16 \\
\hline
\end{tabular}

In addition, there was a 5\% decrease in stroke volume from a heart rate of $147-169$ bpm (Fig 10 (a)). The cardiac output (including pressure differences) increased by $60.2 \%$ from a heart rate of $98-169 \mathrm{bpm}$ (Fig 10(b)). This led to an increment in cardiac output of $5984 \mathrm{ml} / \mathrm{min}$. With considering pressure differences in the 3D FSI models, the discrepancies between echo-Doppler and 3D FSI results notably decreased compared to without considering pressure differences. Consequently, 3D FSI simulation that 
included pressure differences were chosen to continue this study. These results also demonstrated the importance of including the valvular - vascular pressure drops in our study.

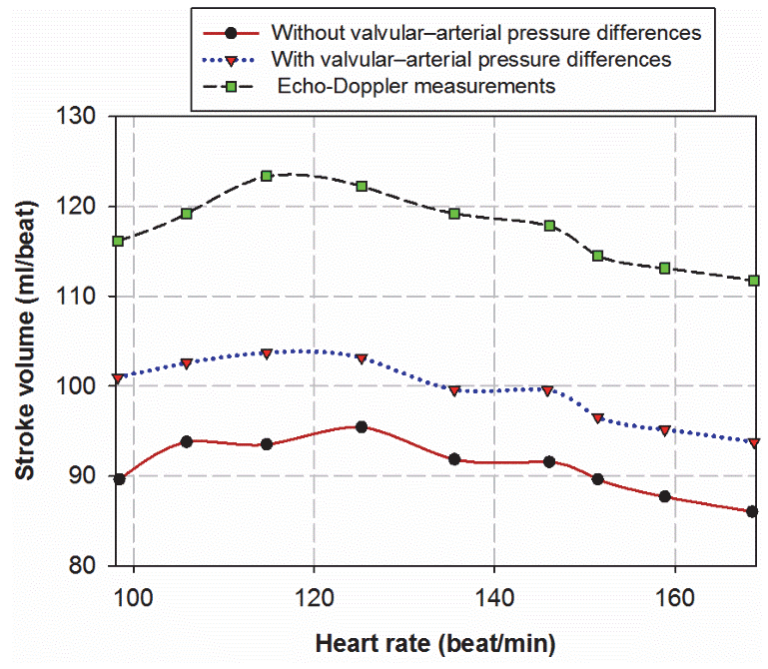

(a)

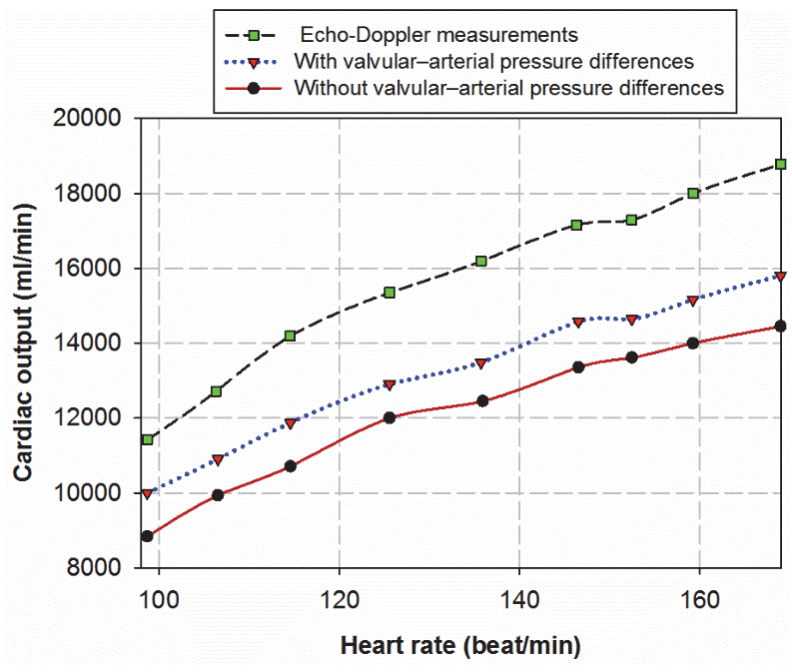

(b)

Fig.10. Comparison of (a) stroke volume and (b) cardiac output among echo-Doppler measurements with and without considering valvular -arterial pressure differences in 3D FSI simulations.

Regression analysis between echo-Doppler and FSI simulations led to a correlation gradient of 0.802 (Fig 11(a)) for cardiac output and 0.764 (Fig. 11(b)) for stroke volume. The Y-axis intercepts for these correlations were $669.1 \mathrm{ml} / \mathrm{min}$ and $15.05 \mathrm{ml} /$ beat for cardiac output and stroke volume, respectively. There was a high correlation between estimations from echo-Doppler and 3D FSI simulations for cardiac output $(\mathrm{r}=0.999)$ and stroke volume $(\mathrm{r}=0.940)$. Therefore, there was a strong correlation between the two methods, and similar values were predicted.

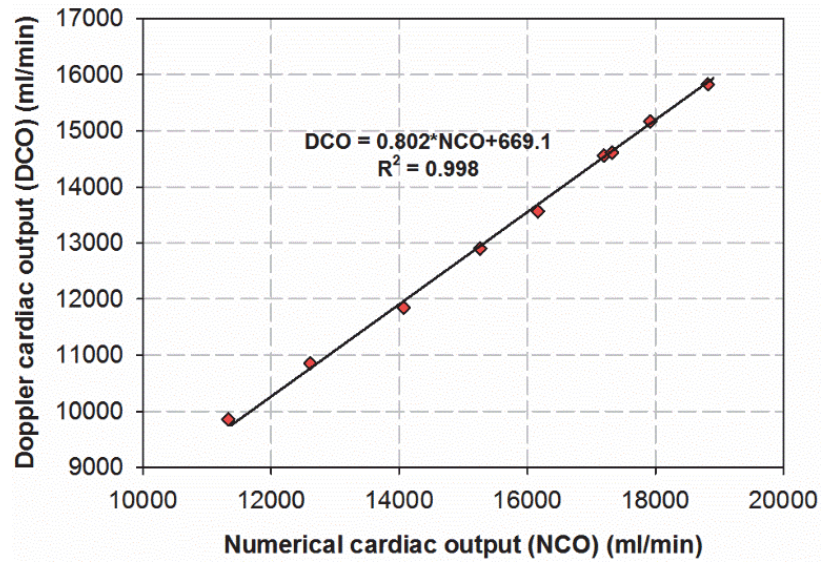

(a)

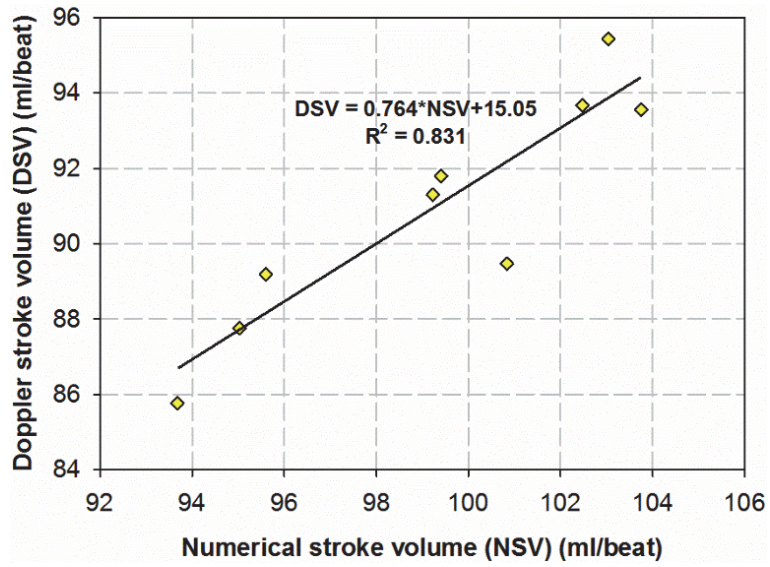

(b)

Fig.11. (a) Regression plot comparing Doppler and numerical cardiac output (DCO) and (NCO) data. (b) Regression plot comparing Doppler and numerical stroke volume (DSV) and (NSV) data.

The relationship between cardiac output and the brachial systolic and diastolic pressure difference is shown in Fig 12. A good correlation was determined using a quadratic polynomial equation for both echo-Doppler and FSI simulations. However, a pressure difference can be estimated between the FSI simulation and the echo-Doppler curve. For instance, echo-Doppler derived cardiac output for the pressure difference of $70 \mathrm{mmHg}$ resulted in $11,356 \mathrm{ml} / \mathrm{min}$. The FSI simulation, instead, estimated a 
cardiac output of $11,356 \mathrm{ml} / \mathrm{min}$ at $87 \mathrm{mmHg}$. This $17-\mathrm{mmHg}$ difference could be due to a valvular and arterial pressure drop. An increase in the brachial pressure difference reduces this pressure drop (Fig 12).

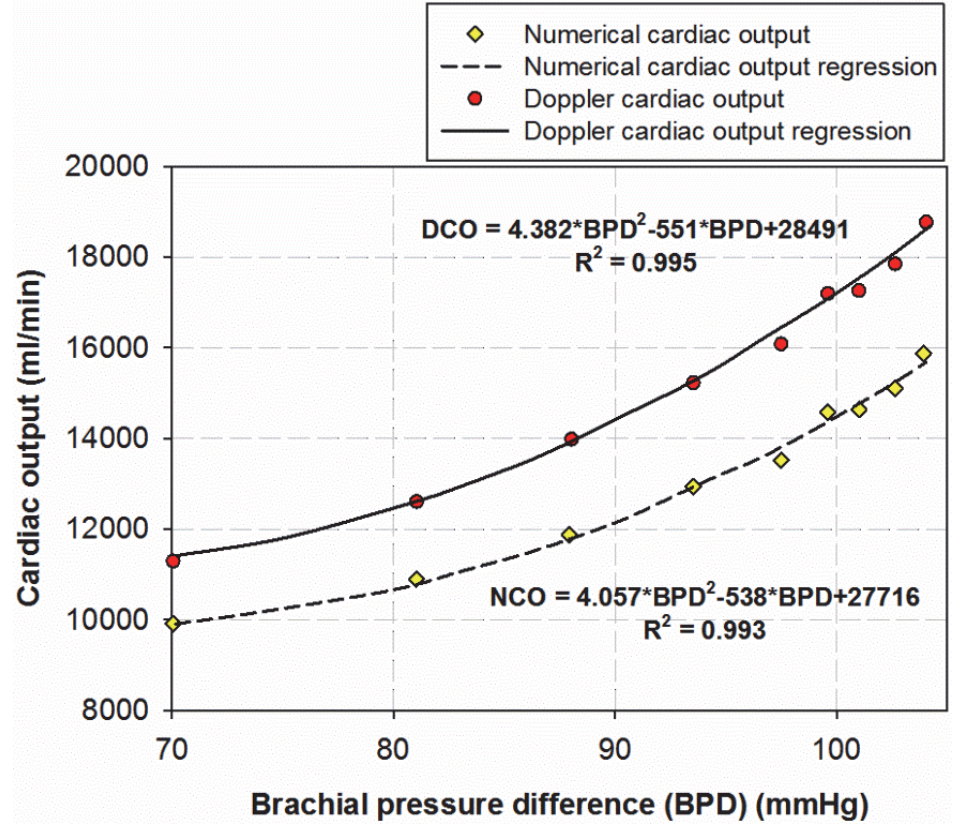

Fig.12. Regression plots of Doppler and numerical cardiac output data versus brachial pressure difference.

The study has combined haemodynamic measurements with an FSI model to non-invasively calculate the cardiac output and stroke volume from a healthy subject during exercise. Echo-Doppler-derived data have been compared to FSI predictions. To our knowledge, this is the first time that an FSI model has been combined with exercise measurements to enable numerical predictions of cardiovascular performance. When valvular - vascular pressure differences were accounted for (stage 1), the predicted cardiac output (using FSI) was lower on average by $2415 \mathrm{ml} / \mathrm{min}$ than Doppler-derived as opposed to $3502 \mathrm{ml} / \mathrm{min}$ when such pressure differences were not accounted for (stage 2). Improved precision of the measured valvular and arterial pressure differences could further reduce the difference between the two methods. Despite the use of a simplified 2D model, FSI predicted values were within $84.6 \%$ of the Doppler-measured values. The FSI model reliably predicted the cardiac output and the mean aortic velocity over a range of heart rates. Predictions of approximately $85 \%$ of experimental measurement would present limitations in clinical use; therefore, linear correlations have been used. This enables highly accurate predictions derived from the FSI model to be obtained (e.g., $\mathrm{r}=0.94$ and 0.999 for stroke volume and cardiac output, respectively). This study demonstrates the feasibility of obtaining a range of time-dependent and variable boundary conditions (e.g., altered due to exercise) and generates a simplified 2D model that can predict cardiovascular performance within a relatively short solution time.

Catheterization thermodilution is the gold standard for measuring the cardiac output (Lavdaniti, 2008). However, it is an invasive method with potential risks such as heart failure, cardiac arrhythmia and even death (Lavdaniti, 2008). In addition, thermodilution exposes the patient and physician to harmful radiation. Exercising while catheterized also causes a range of technical problems, and thus is not common practice. However, the application of a numerical method allows the prediction of cardiac function using non-invasive measurements throughout an exercise protocol. Numerical simulation allows easier and more precise estimation of cardiac output than using echo-Doppler Moreover, it does not have inter- and intra-observer validity variables that are the case when using ECG. Such variability depends on personal proficiency and the image capture capability of the user. Therefore, the key concern is the reliability of numerical methods when predicting the cardiac output. Our FSI model led to a good cardiac output correlation with Doppler-derived values $(\mathrm{r}=0.999)$; besides, a good correlation $(\mathrm{r}=0.94)$ was 
achieved for stroke volume. Data gained when accounting for pressure differences between brachial and central to aortic root at the left ventricle led to differences of $17.9 \mathrm{ml} /$ beat for stroke volume on average. This increased to $26.2 \mathrm{ml} /$ beat when such pressure differences were ignored. There was also a good correlation with the mean velocity $(\mathrm{r}=0.94)$, but the correlation for predicted peak velocity was lower $(\mathrm{r}=0.73)$. However, in the latter case, errors related to Doppler flow tracings may have lowered this correlation. For example, oscillations were observed at the echo-Doppler flow tip, which may have reduced the operator's tracing precision.

\section{Conclusion}

A 3D fluid-structure interaction (FSI) finite element model of the aortic valves and leaflets developed in commercial software (LS-DYNA) which was able to reliably predict the cardiac output and the stroke volume. This computational model predicted mean velocity, stroke volume, and cardiac output within $14.8 \%, 15 \%$ and $15 \%$ of the echo-Doppler measurements. Strong correlations and improvements where achieved by regression equations for predicted and measured cardiac output $(\mathrm{r}=0.999)$ and stroke volume $(\mathrm{r}=0.94)$. The 3D FSI computational model was relatively a rapid tool for clinical assessments, and using its advantages in coupling with clinical measurements demonstrated. The developed 3D FSI computational model was able to be used during both resting and exercising.

\section{Acknowledgement}

The authors the authors wish to thank the anonymous referees for constructive comments on earlier version of this paper.

\section{References}

Al-Atabi, M., Espino, D. M., \& Hukins, D. W. (2010). Computer and experimental modelling of blood flow through the mitral valve of the heart. Journal of Biomechanical Science and Engineering, 5(1), 78-84.

Bellhouse, B. J. (1972). The fluid mechanics of heart valve. Cardiovascular fluid dynamics, 1, 261-285. Caro, C. G. (1978). The systemic microcirculation. The Mechanics of the Circulation, 350-429.

Carmody, C. J., Burriesci, G., Howard, I. C., \& Patterson, E. A. (2006). An approach to the simulation of fluid-structure interaction in the aortic valve. Journal of Biomechanics, 39(1), 158-169.

Christie, J., Sheldahl, L. M., Tristani, F. E., Sagar, K. B., Ptacin, M. J., \& Wann, S. (1987). Determination of stroke volume and cardiac output during exercise: comparison of two-dimensional and Doppler echocardiography, Fick oximetry, and thermodilution. Circulation, 76(3), 539-547.

Clark, R. E., \& Finke, E. H. (1974). Scanning and light microscopy of human aortic leaflets in stressed and relaxed states. The Journal of Thoracic and Cardiovascular Surgery, 67(5), 792.

Criner, G. J., Barnette, R. E., \& D'Alonzo, G. E. (Eds.). (2010). Critical Care Study Guide: Text and Review. Springer Science \& Business Media.

De Hart, J., Peters, G. W., Schreurs, P. J., \& Baaijens, F. P. (2000). A two-dimensional fluid-structure interaction model of the aortic value. Journal of Biomechanics, 33(9), 1079-1088.

De Hart, J., Peters, G. W. M., Schreurs, P. J. G., \& Baaijens, F. P. T. (2003a). A three-dimensional computational analysis of fluid-structure interaction in the aortic valve. Journal of Biomechanics, 36(1), 103-112.

De Hart, J., Baaijens, F. P. T., Peters, G. W. M., \& Schreurs, P. J. G. (2003b). A computational fluidstructure interaction analysis of a fiber-reinforced stentless aortic valve. Journal of Biomechanics, 36(5), 699-712.

Donea, J., Giuliani, S., \& Halleux, J. P. (1982). An arbitrary Lagrangian-Eulerian finite element method for transient dynamic fluid-structure interactions. Computer Methods in Applied Mechanics and Engineering, 33(1-3), 689-723.

Dowell, E. H., \& Hall, K. C. (2001). Modeling of fluid-structure interaction. Annual Review of Fluid Mechanics, 33(1), 445-490. 
Engoren, M., \& Barbee, D. (2005). Comparison of cardiac output determined by bioimpedance, thermodilution, and the Fick method. American Journal of Critical Care, 14(1), 40-45.

Espino, D. M., Shepherd, D. E., \& Hukins, D. W. (2014). Evaluation of a transient, simultaneous, arbitrary Lagrange-Euler based multi-physics method for simulating the mitral heart valve. Computer Methods in Biomechanics and Biomedical Engineering, 17(4), 450-458.

Espino, D. M., Shepherd, D. E., \& Hukins, D. W. (2015). Transient large strain contact modelling: A comparison of contact techniques for simultaneous fluid-structure interaction. European Journal of Mechanics-B/Fluids, 51, 54-60.

Govindarajan, V., Udaykumar, H. S., Herbertson, L. H., Deutsch, S., Manning, K. B., \& Chandran, K. B. (2010). Two-dimensional FSI simulation of closing dynamics of a tilting disk mechanical heart valve. Journal of Medical Devices, 4(1), 011001.

Hofer, C. K., Ganter, M. T., \& Zollinger, A. (2007). What technique should I use to measure cardiac output?. Current Opinion in Critical Care, 13(3), 308-317.

Knobloch, K., Hoeltke, V., Jakob, E., Vogt, P. M., \& Phillips, R. (2008). Non-invasive ultrasonic cardiac output monitoring in exercise testing. International Journal of Cardiology, 126(3), 445-447.

Knobloch, K., Spies, M., Vogt, P. M., \& Phillips, R. (2009). A comparison of real-time CW Doppler and calculated cardiac output according to the Stringer formula for non-invasive hemodynamics in exercise testing. International Journal of Cardiology, 131(3), 413-415.

Koch, T. M., Reddy, B. D., Zilla, P., \& Franz, T. (2010). Aortic valve leaflet mechanical properties facilitate diastolic valve function. Computer Methods in Biomechanics and Biomedical Engineering, 13(2), 225-234.

Laske, A., Jenni, R., Maloigne, M., Vassalli, G., Bertel, O., \& Turina, M. I. (1996). Pressure gradients across bileaflet aortic valves by direct measurement and echocardiography. The Annals of Thoracic Surgery, 61(1), 48-57.

Lavdaniti, M. (2008). Invasive and non-invasive methods for cardiac output measurement. International Journal of Caring Sciences, 1(3), 112.

Maroni, J. M., Oelberg, D. A., Pappagianopoulos, P., Boucher, C. A., \& Systrom, D. M. (1998). Maximum cardiac output during incremental exercise by first-pass radionuclide ventriculography. Chest, 114(2), 457-461.

Murphy, S. L., Xu, J., \& Kochanek, K. D. (2012). Deaths: preliminary data for 2010. National vital statistics reports: from the Centers for Disease Control and Prevention, National Center for Health Statistics, National Vital Statistics System, 60(4), 1-52.

Nobile, F., \& Formaggia, L. (1999). A stability analysis for the arbitrary lagrangian: Eulerian formulation with finite elements. East-West Journal of Numerical Mathematics, 7(EPFL-ARTICLE-176278), 105-132.

Park, S. H., Lee, S. J., Kim, J. Y., Kim, M. J., Lee, J. Y., Cho, A. R., ... \& Jin, D. K. (2011). Direct comparison between brachial pressure obtained by oscillometric method and central pressure using invasive method. Soonchunhyang Medical Science, 17(2), 65-71.

Stringer, W. W., Hansen, J. E., \& Wasserman, K. (1997). Cardiac output estimated noninvasively from oxygen uptake during exercise. Journal of Applied Physiology, 82(3), 908-912.

Stijnen, J. M. A., De Hart, J., Bovendeerd, P. H. M., \& Van de Vosse, F. N. (2004). Evaluation of a fictitious domain method for predicting dynamic response of mechanical heart valves. Journal of Fluids and Structures, 19(6), 835-850.

Sugawara, J., Tanabe, T., Miyachi, M., Yamamoto, K., Takahashi, K., Iemitsu, M., ... \& Matsuda, M. (2003). Non-invasive assessment of cardiac output during exercise in healthy young humans: comparison between Modelflow method and Doppler echocardiography method. Acta physiologica Scandinavica, 179(4), 361-366.

Van de Vosse, F. N., De Hart, J., Van Oijen, C. H. G. A., Bessems, D., Gunther, T. W. M., Segal, A., ... \& Baaijens, F. P. T. (2003). Finite-element-based computational methods for cardiovascular fluidstructure interaction. Journal of Engineering Mathematics, 47(3-4), 335-368. 
Wall, W. A., Gerstenberger, A., Gamnitzer, P., Förster, C., \& Ramm, E. (2006). Large deformation fluidstructure interaction-advances in ALE methods and new fixed grid approaches. In Fluid-structure interaction (pp. 195-232). Springer, Berlin, Heidelberg.

Xia, G. H., Zhao, Y., \& Yeo, J. H. (2005). Numerical simulation of 3D fluid-structure interaction using an immersed membrane method. Modern Physics Letters B, 19(28n29), 1447-1450.

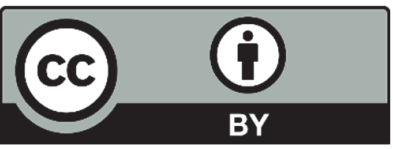

(C) 2020 by the authors; licensee Growing Science, Canada. This is an open access article distributed under the terms and conditions of the Creative Commons Attribution (CC-BY) license (http://creativecommons.org/licenses/by/4.0/). 